\title{
Effects of Pulse Duration of Bulk Laser Damage in 350-nm Raster-Scanned DKDP
}

M. Runkel, J. Bruere, W. Sell, T. Weiland, D. Milam, D. Hahn, M. Nostrand

This article was submitted to

Boulder Damage Symposium XXXIV: Annual Symposium on Optical Materials for High Power Lasers, Boulder, CO, September 16-18, 2002

U.S. Department of Energy

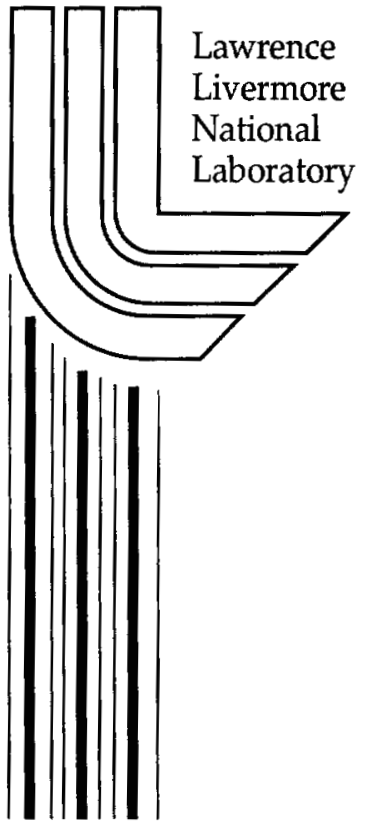

\section{October 30, 2002}




\section{DISCLAIMER}

This document was prepared as an account of work sponsored by an agency of the United States Government. Neither the United States Government nor the University of California nor any of their employees, makes any warranty, express or implied, or assumes any legal liability or responsibility for the accuracy, completeness, or usefulness of any information, apparatus, product, or process disclosed, or represents that its use would not infringe privately owned rights. Reference herein to any specific commercial product, process, or service by trade name, trademark, manufacturer, or otherwise, does not necessarily constitute or imply its endorsement, recommendation, or favoring by the United States Government or the University of California. The views and opinions of authors expressed herein do not necessarily state or reflect those of the United States Government or the University of California, and shall not be used for advertising or product endorsement purposes.

This is a preprint of a paper intended for publication in a journal or proceedings. Since changes may be made before publication, this preprint is made available with the understanding that it will not be cited or reproduced without the permission of the author.

This work was performed under the auspices of the United States Department of Energy by the University of California, Lawrence Livermore National Laboratory under contract No. W-7405-Eng-48.

This report has been reproduced directly from the best available copy.

Available electronically at http://www.doc.gov/bridge

Available for a processing fee to U.S. Department of Energy

And its contractors in paper from

U.S. Department of Energy

Office of Scientific and Technical Information

P.O. Box 62

Oak Ridge, TN 37831-0062

Telephone: (865) 576-8401

Facsimile: (865) 576-5728

E-mail: reports@adonis.osti.gov

Available for the sale to the public from

U.S. Department of Commerce

National Technical Information Service

5285 Port Royal Road

Springfield, VA 22161

Telephone: (800) 553-6847

Facsimile: (703) 605-6900

E-mail: orders@ntis.fedworld.gov

Online ordering: http://www.ntis.gov/ordering.htm

OR

Lawrence Livermore National Laboratory

Technical Information Department's Digital Library

http://www.llnl.gov/tid/Library.html 


\title{
Effects of pulse duration on bulk laser damage in 350-nm raster- scanned DKDP
}

\author{
Mike Runkel, Justin Bruere, Wally Sell, Tim Weiland, David Milam, Doug Hahn, Mike Nostrand \\ Lawrence Livermore National Laboratory \\ P. O. Box 808, L-491, Livermore, CA 94551 \\ (925)-424-2210, runkell@llnl.gov
}

\begin{abstract}
In this paper we present the results of bulk damage experiments done on Type-II DKDP triple harmonic generator crystals that were raster conditioned with 351-355 nm wavelengths and pulse durations of 4 and $23.2 \mathrm{~ns}$. In the first phase of experiments 20 different scan protocols were rastered into a sample of rapid growth DKDP. The sample was then rastered at damage-causing fluences to determine the three most effective protocols. These three protocols were scanned into a $15-\mathrm{cm}$ sample of conventional-growth DKDP and then exposed to single shots of a $1-\mathrm{cm}$ beam from LLNL's Optical Sciences Laser at fluences ranging from $0.5-1.5 \mathrm{X}$ of the $10 \%$ damage probability fluence and nominal pulse durations of $0.1,0.3,0.8,3.2,7.0$ and $20 \mathrm{~ns}$. The experiment showed that pulse durations in the 1-3 ns range were much more effective at conditioning than pulses in the $16.3 \mathrm{~ns}$ range and that the multiple pass "peak fluence" scan was more effective than the single pass "leading edge" scan for $23.2 \mathrm{~ns}$ XeF scans.
\end{abstract}

\section{INTRODUCTION}

Potassium dihydrogen phosphate (KDP) and its deuterated isomorph (DKDP) are the crystals of choice for the next generation of inertial confinement (ICF) fusion lasers. This is because the crystals can be grown large enough to provide 40-cm clear aperture plates for Pockel's cells and frequency conversion crystals. Each succeeding generation of ICF laser has operated at higher average fluence with full fluence shots for the National Ignition Facility (NIF) planned to be $8 \mathrm{~J} / \mathrm{cm}^{2}$ at $351 \mathrm{~nm}$ in a $3 \mathrm{~ns}$ pulse. To meet the demands of increased fluence, the damage resistance of KDP and DKDP crystals, particularly third harmonic generators (THG), has also increased ${ }^{1}$. Extensive efforts in growing crystals with less particulate and elemental contaminants have resulted in significantly more damage resistant crystals for the next generation of ICF lasers. We now believe that current crystals will be able to survive laser shots up to $4 \mathrm{~J} / \mathrm{cm}^{2}(1.3$ $\mathrm{GW} / \mathrm{cm}^{2}$ ) without conditioning. In order to meet damage specifications for higher fluence shots, the THG crystals will require laser conditioning to increase damage resistance. Significant on-line conditioning is expected to occur during activation of individual beamline ${ }^{2}$ but there are concerns regarding uniformity, risk to optics from aberrant shots and the cost of firing the laser. In addition replacement crystals may need to be installed during high fluence shot campaigns where a series of conditioning shots is not feasible. In the case of either beamline activation or operations, it is desirable to deliver a uniformly conditioned crystal to the ICF laser. Recently we have concentrated on developing laser raster conditioning using a variety of tripled Nd:YAG and excimer lasers and pulse durations ${ }^{3,4,5}$. Laser conditioning at 1064 $\mathrm{nm}(1 \omega)$ is already being used for conditioning of large mirrors and polarizers ${ }^{6}$ but UV ( 351 or $\left.355 \mathrm{~nm}, 3 \omega\right)$ laser conditioning is required for DKDP triplers. To this end we have focused on developing a laser raster scanning station based on commercially available table-top Nd:YAG and excimer laser systems which is described below. In the first experiments reported here, our goal was to use this station, known as Phoenix, to determine the optimal conditioning protocols for each laser. In the second phase, our goal was to verify the effectiveness of the conditioning protocols in a large $(\mathrm{cm})$ beam environment over pulse durations expected to be used on NIF (0.1-20 ns). For this, LLNL's Optical Sciences Laser (OSL) was ideal.

\section{THE PHOENIX CONDITIONING LABORATORY}

The Phoenix conditioning laboratory consists of a $50 \mathrm{~Hz}$, tripled Nd:YAG laser with $3.7 \mathrm{~ns}$ pulse duration and far field $1 / \mathrm{e}^{2}$ beam diameter of $1.2 \mathrm{~mm}$. This beam is formed using a 2 element telescope with effective focal length of $\sim 6 \mathrm{~m}$. A fused silica wedge is used to split off diagnostic beams for energy, temporal and spatial profile characterization. This 
layout is common to other LLNL damage test systems. The maximum fluence attainable with this system is $\sim 50 \mathrm{~J} / \mathrm{cm}^{2}$. In addition to the Nd:YAG, a $100 \mathrm{~Hz}$, XeF laser with 23 ns pulse duration was installed in the lab. This laser uses a single-axis beam homogenizer to deliver a rectangular gaussian top hat beam where the gaussian short axis has $1 / \mathrm{e}^{2}$ diameter of $0.5 \mathrm{~mm}$ and the top-hat, long axis has FWHM of $1.5 \mathrm{~mm}$. It can deliver beam fluences up to $50 \mathrm{~J} / \mathrm{cm}^{2}$. The $\mathrm{Nd}$ :YAG and excimer beams are delivered to a 2-axis stage capable of holding a full-sized 40-cm optic and positioning it to within a few microns. Visible laser diodes are available to color the UV beams for in-situ scatter mapping and longworking distance microscopes can be used for post-scan inspection of the sample at magnifications up to 110X. The primary diagnostic is known as the Damage Mapping System (DMS) ${ }^{\top}$. This consists of a line-scan CCD camera that is used to take darkfield images of the entire optic. The test samples are mounted so that light bars may be used to provide side illumination for highly sensitive darkfield imagery. The beam fluence is set manually with either laser system, and the stage and diagnostics are computer controlled.

\section{ISSUES WITH USING GAUSSIAN BEAMS IN RASTER SCANNING}

In producing up to $200,40-\mathrm{cm}$, uniformly conditioned tripler crystals, a balance must be achieved between system throughput and degree of conditioning. For the NIF, the expected maximum demand for conditioned triplers is five per week (one/day). This sets the average laser power requirement for DKDP triplers to be $\sim 20$ Watts (see reference 5). This power level is readily achievable using commercially available table-top laser systems and the two candidates are tripled Nd:YAG and excimer. From purely a scan efficiency standpoint, a square top-hat beam would be optimal followed by round top-hat, then beams with gaussian components. However, for the Nd:YAG case, constructing a tophat beam delivery system is substantially more involved than delivering a far-field gaussian beam. For the excimer, beam homogenization can deliver high quality square top-hat or gaussian beams with little difficulty. Since there is a substantial scan time penalty for using beams with a gaussian spatial component, the deciding factor for their usage is whether the peak fluence or the leading edge of the beam contributes most to conditioning. In [2] we showed that conditioning could be achieved in as few as four shots with the OSL beam at succeedingly higher fluences and that more shots (smaller fluence steps) gave better conditioning. In addition, unpublished LLNL results have shown that 10 rapid scans with peak fluence steps of $1 \mathrm{~J} / \mathrm{cm}^{2}$ between scans have been successful in conditioning some DKDP triplers. The rapid scan rate is also thought to reduce the occurrence of surface damage "dragging" should it initiate in the later, high fluence passes. In contrast, previous work with excimer lasers ${ }^{4}$ shows that significant conditioning is achievable with a slow, single pass scan to high fluence where the gaussian leading edge of the beam acts as the fluence ramp. The "peak fluence" and "leading edge" scan protocols are shown in Figure 1.

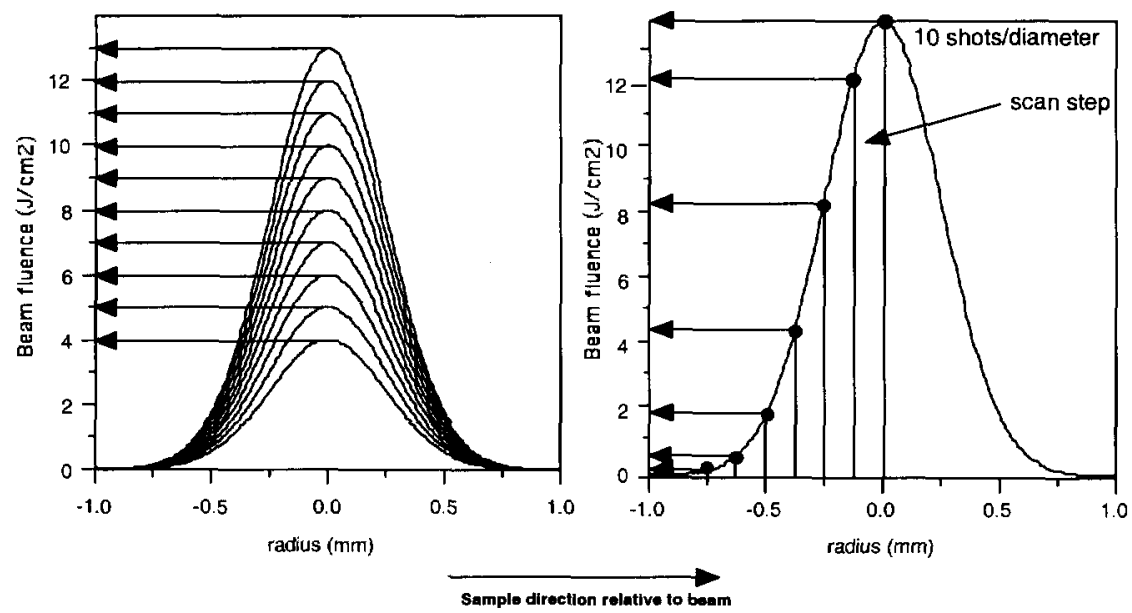

Figure 1. Peak fluence (left) and leading edge (right) raster scan protocols. For peak fluence, each scan is done rapidly with beam overlap not exceeding $90 \%$. The fluence is incremented by $1 \mathrm{~J} / \mathrm{cm}^{2}$ between scans. Conditioning as high as $14 \mathrm{~J} / \mathrm{cm}^{2}, 3.2 \mathrm{~ns}$ has been achieved with this method on conventional-growth crystals. For the leading edge a single scan is done slowly to the peak fluence and the leading edge of the beam provides conditioning. Beam overlaps exceed $99 \%$ for leading edge scans. 


\section{PHASE 1 EXPERIMENTS - DOWNSELECTING SCAN PROTOCOLS}

\subsection{Sample selection}

The material of main interest for these tests is conventionally grown, 70\% deuterated, type II tripler cuts of DKDP. The current state-of-the-art material was grown by Cleveland Crystals, Inc. and at the time of these tests was represented by boule LL11. This was the one of the first NIF production boules to reach yield. Consequently, several $15-\mathrm{cm}$ pieces were available for testing. While available for use, these samples were not plentiful so it was decided to use a comparable piece of rapid-growth material for downselecting the raster scan protocols. This material was obtained from boule CD41. Damage tests using the Zeus laser at $3 \omega, 8$ ns on witness samples from the two boules showed that unconditioned $(S / 1)$ and conditioned $(R / 1)$ damage probability curves were essentially identical. This is shown in Figure 2.

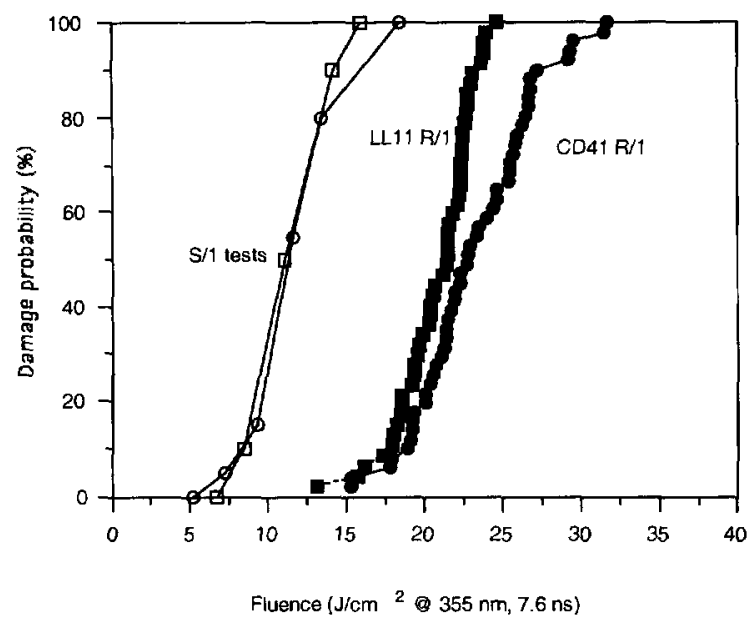

Figure 2. Damage probability curves from CD41 and LL11 showing that the damage performance of the two boules is essentially identical.

\subsection{Test plan for CD41 raster-scan protocol downselect}

In order to determine the most effective scan protocols CD41 was raster scanned with 12 scan protocols of both peakand leading-edge types using the XeF laser ( $23 \mathrm{~ns})$. In addition, the Nd:YAG laser $(4 \mathrm{~ns})$ was used to perform a peak fluence scan to determine the highest level of conditioning attainable for the crystal. Each scan region was $1.5 \mathrm{X} 1.5$ $\mathrm{cm}^{2}$. After all protocols were scanned into the crystal, a DMS map was taken to evaluate bulk damage that might have occurred during the scans. After this, the Nd:YAG laser was set to $8 \mathrm{~J} / \mathrm{cm}^{2}$ to guarantee bulk damage in unconditioned material and a single scan was made over the entire region with $50 \%$ beam overlap. This ensured that the damage scan did not condition the sample. After this, the sample was again DMS mapped and the most damage resistant protocols were determined from the DMS map, visual inspection and microscopy. The most effective leading edge and peak fluence protocols were chosen from the $\mathrm{XeF}$ while the peak fluence protocol would be used for the Nd:YAG laser. Table 1 lists the various scan protocols. 
Table 1. Peak fluence and leading edge scan protocols using Nd:YAG and XeF lasers for CD41. The table is grouped according to pulse duration with 4 ns protocols being first. Protocols 5, 16 and 17 were chosen for large beam damage testing and are indicated with boldface type.

\begin{tabular}{|c|c|c|c|c|c|}
\hline Number & Protocol & Pulse length (ns) & Beam overlap & Passes & $\begin{array}{l}\text { Fluence (no t- } \\
\text { scaling) }\end{array}$ \\
\hline 1 & Peak fluence & 4 & 0.9 & 7 & $4.3-11.0$ \\
\hline 2 & Peak fluence & 4 & 0.95 & 7 & $4.3-11.0$ \\
\hline 17 & Peak fluence & 4 & 0.5 -four pass & 6 - four pass & $4.4-9.9$ \\
\hline 18 & Peak fluence & 4 & 0.5 -four pass & $6-$ four pass & $4.4-9.9$ \\
\hline 19 & Repeat 17 & 4 & 0.5 -four pass & 6 - four pass & $4.4-9.9$ \\
\hline 20 & Repeat 18 & 4 & 0.5 -four pass & 6 - four pass & $4.4-9.9$ \\
\hline 21 & Peak fluence & 4 & 0.5 & 1 & 11.0 \\
\hline 22 & Peak fluence & 4 & 0.5 & 1 & 8.8 \\
\hline 23 & Peak Fluence & 4 & 0.5 & 1 & 6.6 \\
\hline 3 & Peak fluence & 23 & 0.9 & 3 & $8.2-24.8$ \\
\hline 4 & Peak fluence & 23 & 0.9 & 7 & $6.7-24.8$ \\
\hline 5 & Peak fluence & 23 & 0.9 & 11 & $7.1-24.8$ \\
\hline 6 & Leading edge & 23 & 0.94 & 11 & $7.1-24.8$ \\
\hline 7 & Leading edge & 23 & 0.99 & 11 & $7.1-24.8$ \\
\hline 9 & Leading edge & 23 & 0.9 & 1 & 24.8 \\
\hline 10 & Leading edge & 23 & 0.994 & 1 & 24.8 \\
\hline 11 & Leading edge & 23 & 0.9999 & 1 & 24.8 \\
\hline 12 & Leading edge & 23 & 0.9994 & 1 & 24.8 \\
\hline 13 & Leading edge & 23 & 09998 & 1 & 24.8 \\
\hline 15 & Leading edge & 23 & 0.9993 & 1 & 31.0 \\
\hline 16 & Leading edge & 23 & 0.998 & 1 & 31.0 \\
\hline
\end{tabular}

Figure 3 shows the DMS map for the sample after the conditioning and damage scans were performed.

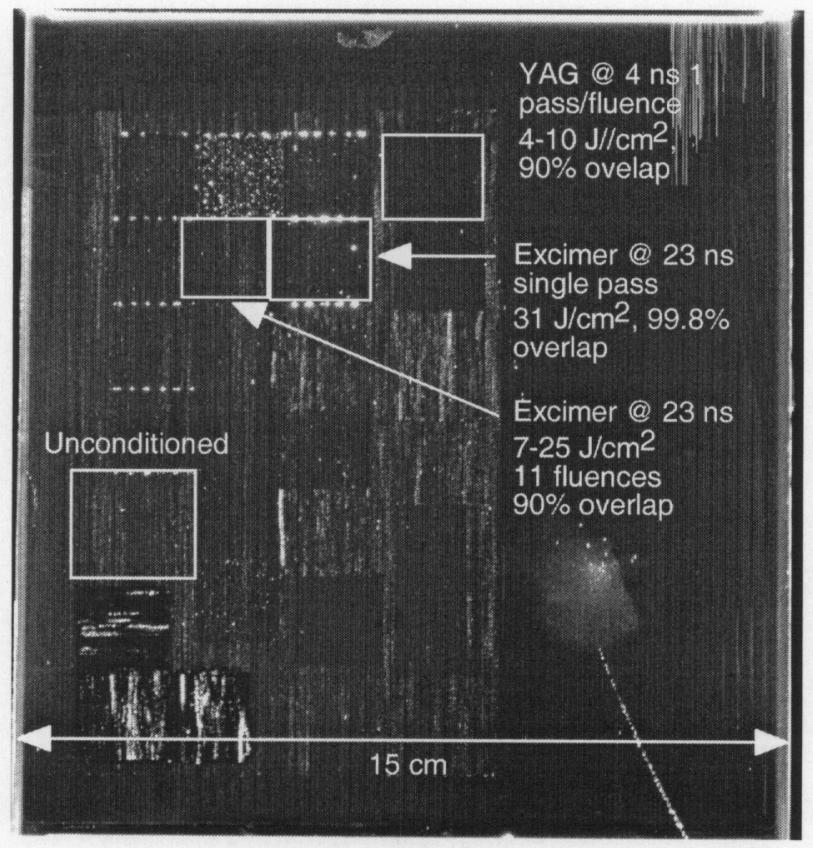

Figure 3. Damage map of CD41 after conditioning and damage scans were performed. The boxes indicate the difference between unconditioned and laser conditioned regions. The image also shows a checkerboard like pattern where other (less effective) protocols were rastered, vacuum chuck lines and some bulk damage at the beginning of excimer scan lines. 
The DMS map shows numerous features including vacuum chuck lines that appear as vertical stripes over the entire sample. In addition, the map shows a checkerboard-like pattern where other protocols show conditioning. There are three grid squares in the lower left that show surface cleaning and substantial bulk damage. These were performed when the Nd:YAG laser was not stable due to injection seeder misalignment and were rejected. Also, the excimer-conditioned regions typically exhibit bulk damage at the beginning of scan lines where the sample was exposed to high fluence initially (in the leading edge scans). This damage stops as a result of leading edge conditioning. Inspection of the part indicated that scan protocols 5 and 16 for the XeF laser and scan protocol 17 for the Nd:YAG laser were most effective against the $8 \mathrm{~J} / \mathrm{cm}^{2}$, Nd:YAG damage scan. The $31 \mathrm{~J} / \mathrm{cm}^{2}, 23.2 \mathrm{~ns}$ peak fluence protocol 16 induced a few bulk pinpoints while the $24.8 \mathrm{~J} / \mathrm{cm}^{2}$ peak fluence of the 11-pass peak fluence scan did not, so we decided to raster up to $28 \mathrm{~J} / \mathrm{cm}^{2}, 23 \mathrm{~ns}$ for the LL11 scans of phase 2.

\section{PHASE 2 EXPERIMENTS - OSL TESTS OF CONDITIONING PROTOCOLS}

\subsection{Damage test plan for sample LL11}

Phase 2 of these experiments was to determine the effectiveness of the three, downselected raster conditioning protocols in a large beam environment. The test plan called for scanning the protocols into 3 of $4,6 \times 6 \mathrm{~cm}^{2}$ quadrants of a $15 \mathrm{X}$ $15 \mathrm{X} 1 \mathrm{~cm}^{3}$ sample of conventional growth LL11, then exposing the conditioned material to single 351-nm pulses from the 1-cm diameter Optical Sciences Laser with pulse durations of 0.1, 0.3, 1, 3, 10 and $16.3 \mathrm{~ns}$ and average fluences of $0.5,0.8,1.0,1.5$ and 1.5 times the S/1 $10 \%$ damage probability fluence (see Figure 2). The same shot sequence would be fired on the remaining, unconditioned quadrant as a control giving a total of 100 shots on the sample. This is shown pictorially in Figure 4.

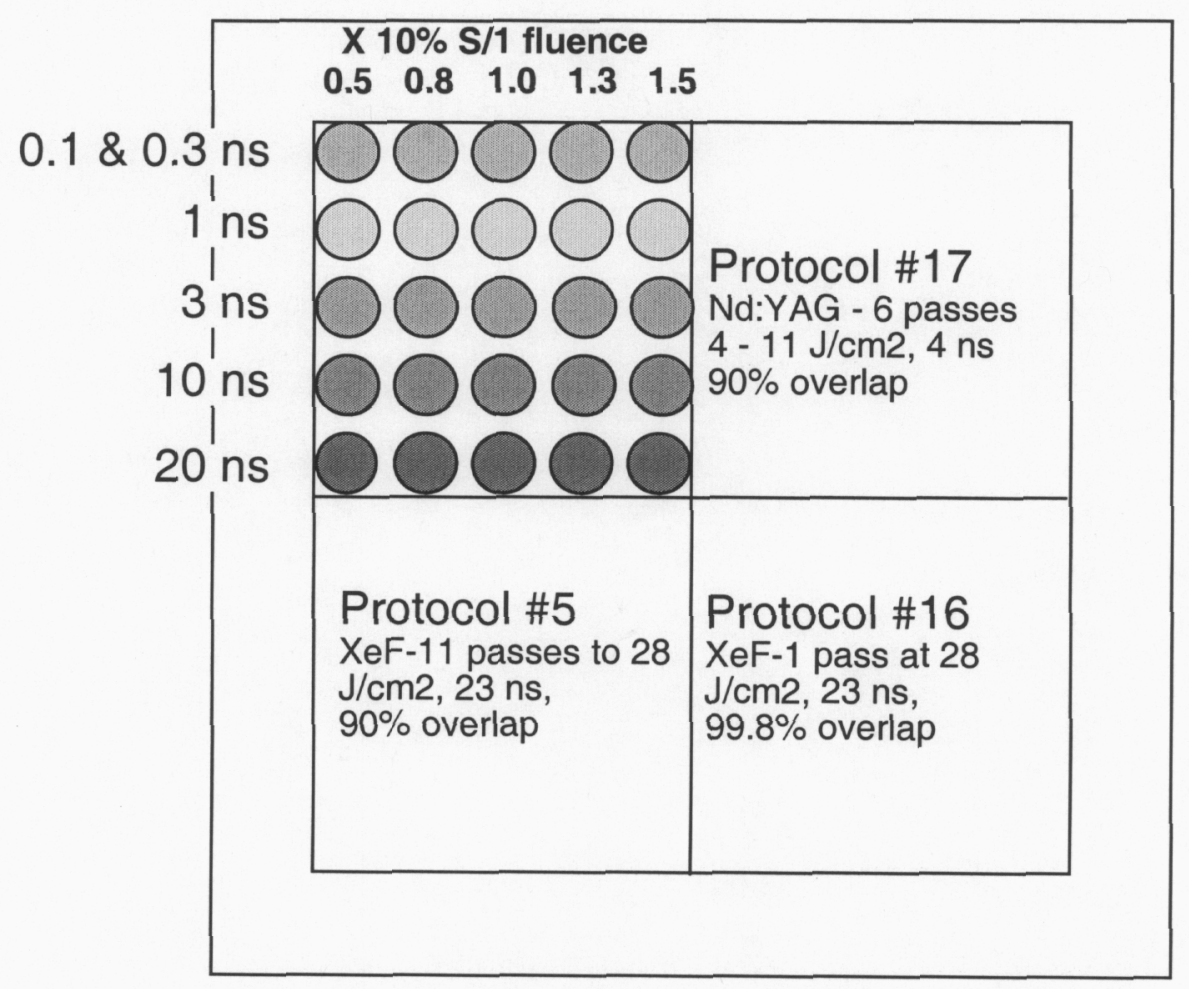

Figure 4. Schematic of the OSL shot plan for testing the raster conditioning protocols from the phase 1 tests. The upper left quadrant was not conditioned. The remaining three quadrants were rastered with protocols 5 and 16 (XeF, $23 \mathrm{ns)}$ and protocol \#17 (Nd:YAG, 4 ns), then exposed to nominally identical shots as the unconditioned quadrant.

After the OSL shots, the sample was examined with the DMS mapping system, visual inspection and microscopy to determine the effectiveness of the conditioning protocols at the different pulse durations and fluences. 


\subsection{The Optical Sciences Laser beam}

The Optical Sciences Laser at LLNL is a Nd:Glass laser capable of producing hundreds of joules of $1053 \mathrm{~nm}$ light per pulse. This light was frequency converted to 351-nm using a Type II/Type II conversion scheme and KDP/DKDP crystals. A 1-cm diameter near flat-top spatial profile was produced by passing the beam through an aperture prior to frequency conversion, then imaging the output of the triple harmonic generation crystal onto the sample plane. The diameter of the flat-top portion of the beam was nominally $1 \mathrm{~cm}$. When falloff from the wings of the beam was considered, the base diameter extended to $1.5 \mathrm{~cm}$. To achieve effective frequency conversion over pulse durations ranging from 0.1 to $16.3 \mathrm{~ns}$, two sets of crystals were used. The range of pulse durations was achieved by using a pulse slicer to produce nominally square temporal profiles. The variability of the pulse durations and shapes could be significant from shot-to-shot because of timing jitter of the pulse slicer and the steeply rising amplifier gain curve. Typical spatial and temporal profiles from a $16.3 \mathrm{~ns}$ shot are shown if Figure 5. Post shot analysis showed average pulse durations of $16.3,7.0,3.2$ and $0.8 \mathrm{~ns}$ for the $20,10,3$ and $1 \mathrm{~ns}$ settings from the test plan. The beam contrast was 1.15:1.
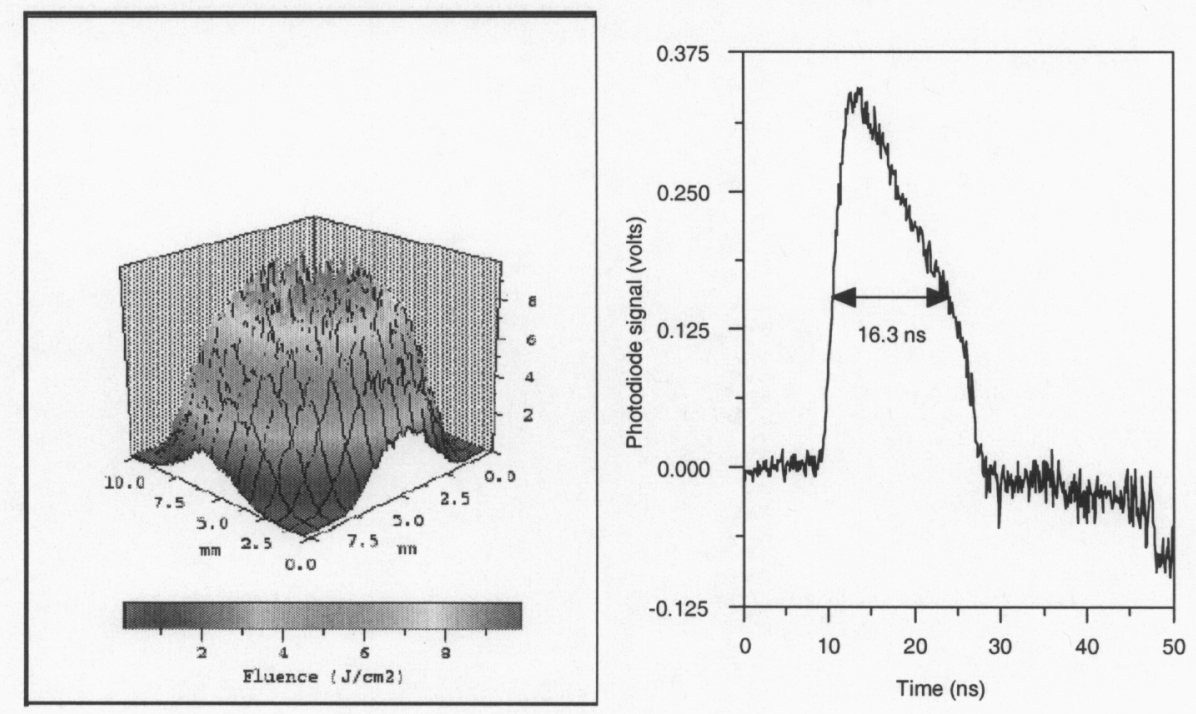

Figure 5. Spatial and temporal profiles of a 16.3-ns OSL shot at the sample plane. The nominal diameter of the flat-top portion is 1 $\mathrm{cm}$ and this portion has rms contrast of 15 percent while the base of the beam has diameter of $\sim 1.5 \mathrm{~cm}$.

\subsection{Results of OSL shots}

Figure 6 shows the DMS map taken after all OSL shots had been completed.

The left hand image in Figure 6 shows the DMS map under maximum lighting intensity. The right hand image is the same map with an grid overlay showing the average fluence from the flat-top portion of the beam. Also indicated are the nominal pulse durations for the shots. The upper left quadrant was not laser conditioned. The upper right quadrant was the 6-pass Nd:YAG scan to $11 \mathrm{~J} / \mathrm{cm}^{2}, 4$ ns. The lower right quadrant was the single pass XeF scan to $28 \mathrm{~J} / \mathrm{cm}^{2}, 23 \mathrm{~ns}$ while the lower left quadrant was the 11-pass XeF scan to $28 \mathrm{~J} / \mathrm{cm}^{2}, 23 \mathrm{~ns}$. The pulse durations were changed as indicated by the numbers between the two maps. The $16.3 \mathrm{~ns}$ shots were performed first in the top row of each quadrant. These were followed by a single shot at $0.1 \mathrm{~ns}$ in the first column, bottom row, of each quadrant. The remaining four sites of these rows were exposed to nominally $0.3 \mathrm{~ns}$ pulses. The fourth row from the top in each quadrant was then exposed to $0.8 \mathrm{~ns}$ pulses followed by the third row at $7.0 \mathrm{~ns}$, then the second row at $3.2 \mathrm{~ns}$. 

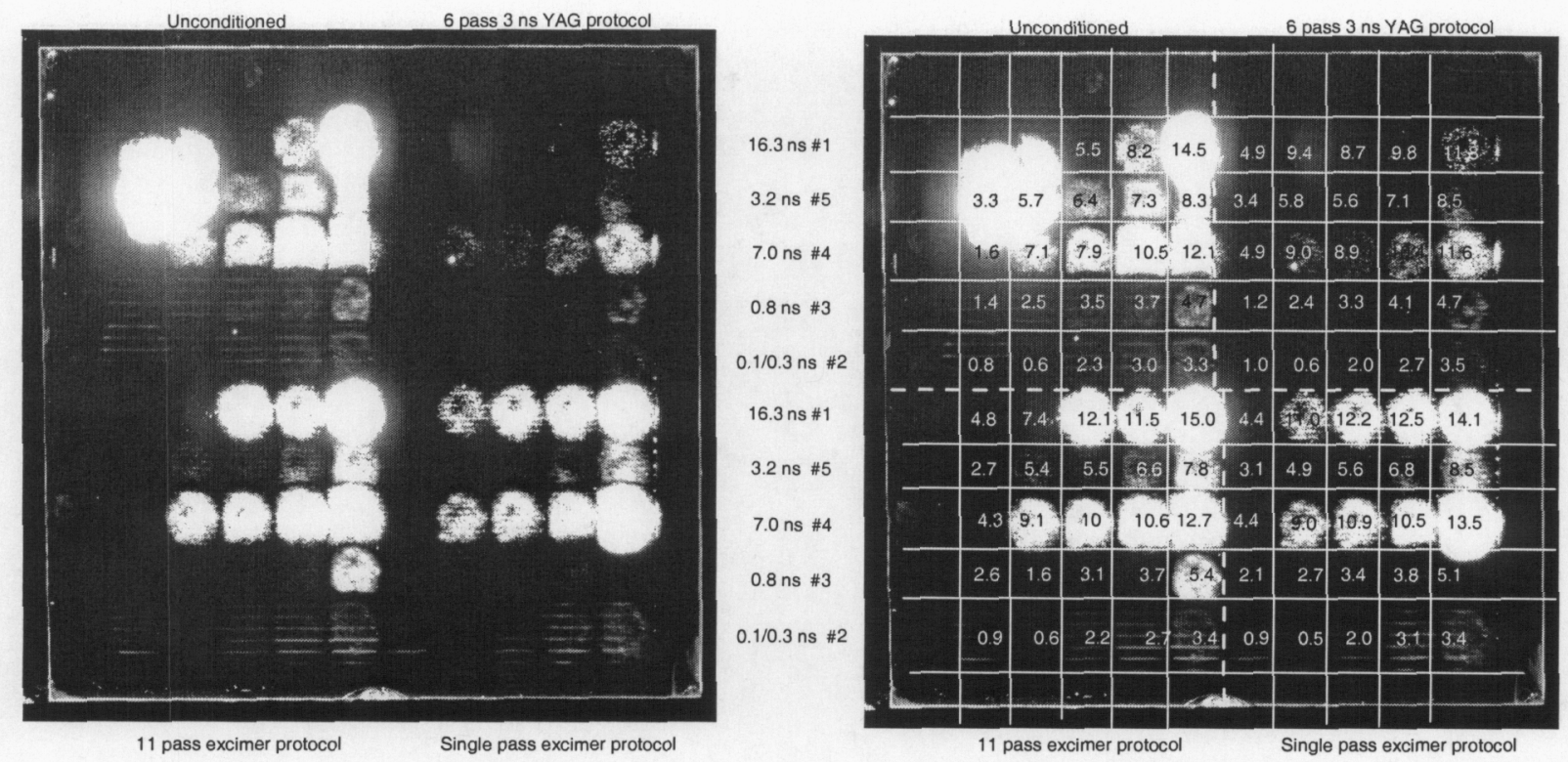

Figure 6. DMS map of LL11 sample after exposures to single shots at $351 \mathrm{~nm}$ on OSL. The left image shows the sample with maximum lighting level while the right is the same image with a grid overlay giving average fluence of the shot.

Inspection of the DMS map reveals several things. The large damage site in the unconditioned quadrant was due to several high fluence shots at $16.3 \mathrm{~ns}$ being overlaid because the sample was translated only $1 \mathrm{~mm}$ between pulses rather than the intended $1 \mathrm{~cm}$. This was detected early in testing and once corrected had little impact on the rest of the experiments. Next, in what is the main point of this work, the DMS map shows that damage at all pulse durations and fluences was less severe in the Nd:YAG conditioned quadrant. This indicates that the 4 ns pulse duration is more effective at bulk conditioning than 23 ns. This trend also appears to hold for shorter pulses as well. Inspection of the unconditioned quadrant shows that the single pulses of shorter pulse durations effectively condition for longer pulses. This is particularly evident for the $0.8 \mathrm{~ns}$ and $7.0 \mathrm{~ns}$ rows as shown in Figure 7.

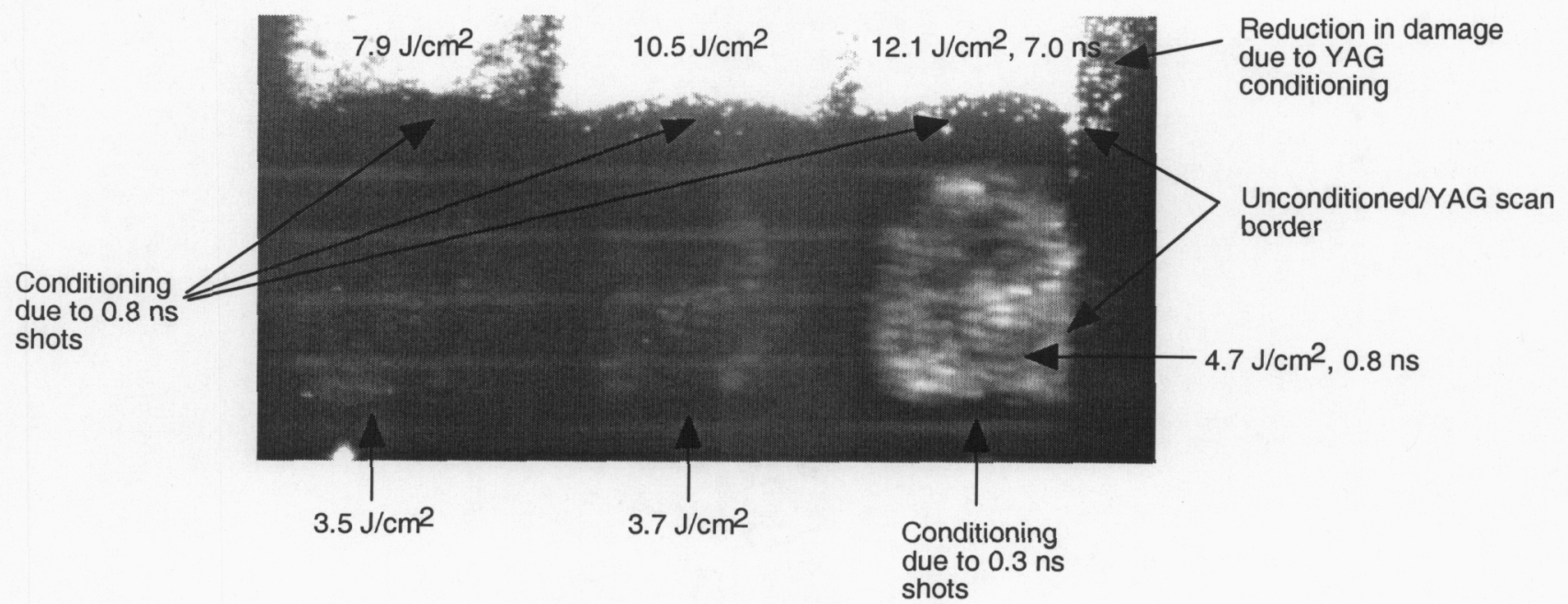

Figure 7. A section of the DMS map from Figure 6 showing bulk damage from the $7.0 \mathrm{~ns}$ row (top) and the $0.8 \mathrm{~ns}$ row (bottom). The effects of the $4 \mathrm{~ns}$ Nd:YAG conditioning are evident from the vertical border on the right side of the image. The effects of single pulse conditioning from beam overlap are evident by the circular edges on the $7.0 \mathrm{~ns}$ sites. Conditioning from the $0.3 \mathrm{~ns}$ row is also evident at the bottom of the $4.7 \mathrm{~J} / \mathrm{cm}^{2}, 0.8 \mathrm{~ns}$ site. 
In Figure 7 the boundary between the unconditioned and Nd:YAG conditioned region appears as a vertical edge. A substantial reduction in bulk pinpoint density can be seen for both the $7.0 \mathrm{~ns}$ and $0.8 \mathrm{~ns}$ regions. In addition, the effect of single pulse conditioning on the $7.0 \mathrm{~ns}$ sites due to beam overlap from the $0.8 \mathrm{~ns}$ sites is evident in the top row of damage sites. Here the border between unconditioned and conditioned regions takes the form of a hard circular edge from beam overlap. This can also be seen for the $4.7 \mathrm{~J} / \mathrm{cm}^{2}, 0.8 \mathrm{~ns}$ shot that was conditioned by beam overlap from the $3.3 \mathrm{~J} / \mathrm{cm}^{2}, 0.3 \mathrm{~ns}$ shot. In addition, it is apparent from the image that the size of the pinpoints is much larger for the 7.0 ns shots. This repeats the observation of [8] where, as a function of beam fluence, it was discovered that shorter pulse produce more and smaller bulk damage sites.

Further inspection of the DMS map in Figure 6 shows that bulk damage is not entirely eliminated for either the 4 ns conditioning pulse length when shot with similar pulse duration and fluence. This can be seen for the $4 \mathrm{~ns}$ scan quadrant for the $8.5 \mathrm{~J} / \mathrm{cm}^{2}$ shot. Analysis of the fluence profile for this shot shows that less than $0.05 \%$ of the beam fluence exceeded the $11 \mathrm{~J} / \mathrm{cm}^{2}$ raster scan fluence and this cannot account for the amount of bulk damage seen at the test site. In the 4-ns Nd:YAG scan quadrant, it is likely that there was significant underscanning due to far-field beam wander from the laser source. We explored the effect of beam wander for this laser in [5] and found that for the $300 \mu \mathrm{m}$ beam wander and $\pm 15 \%$ fluence fluctuations, a scan with $90 \%$ beam overlap would cover the optic with $70 \%$ of the desired peak fluence or less. In reality, the $100 \%$ coverage fluence for the quadrant was probably about $7.7 \mathrm{~J} / \mathrm{cm}^{2}$. For comparison, the $7.1 \mathrm{~J} / \mathrm{cm}^{2}, 3.2 \mathrm{~ns}$ shot in the Nd:YAG quadrant shows a much lower degree of bulk damage than the neighboring 8.5 $\mathrm{J} / \mathrm{cm}^{2}$ shot. The small number of bulk pinpoints seen in the $7.1 \mathrm{~J} / \mathrm{cm}^{2}$ shot can be accounted for by high-fluence portions of the beam.

A similar lack of conditioning holds for both XeF raster scan regions when shot at 16.3 ns. For example, no portion of the $14.1 \mathrm{~J} / \mathrm{cm}^{2}, 16.3 \mathrm{~ns}$ OSL shot exceeded the $23 \mathrm{~ns}$ raster scan fluence of $28 \mathrm{~J} / \mathrm{cm}^{2}$. This also holds for the $15.0 \mathrm{~J} / \mathrm{cm}^{2}$ OSL shot in the 11-pass XeF quadrant. This cannot be so readily explained using beam wander and fluence fluctuations because the XeF laser was substantially more stable than the Nd:YAG with fluence fluctuations off $\pm 5 \%$ and beam wander of less than 50 microns. In addition, the beam overlaps for the two protocols were $90 \%$ and $99.8 \%$. The beam ripple in the latter case would be approximately $0.2 \%$. We are therefore left to conclude that conditioning with the XeF laser was incomplete. One reason for this was the $\sim 1 \mathrm{~cm}$ focal waist of the beam homogenization system may not have been accurately positioned to focus in the center of the test piece. With optimal positioning the fluence on the surfaces would only be $15 \%$ lower than in the center. In the extreme case of having the focus of the beam on the rear surface, the fluence on the front surface would be 1.7 times lower. While it is certain that some part of the crystal did see the peak fluence of $28 \mathrm{~J} / \mathrm{cm}^{2}$ for the XeF scans, the average fluence throughout the bulk in this case would be approximately $75 \%$ of peak or $21 \mathrm{~J} / \mathrm{cm}^{2}$. Still, this is not enough to account for the bulk damage in the $16.3 \mathrm{~ns}$ rows. Thus we need to look for other reasons for the lack of conditioning. One possibility is that conditioning is irradiance dependent. Note that the $9.9 \mathrm{~J} / \mathrm{cm}^{2}$ peak fluence of the $4 \mathrm{~ns}\left(3.7\right.$ in actuality) Nd:YAG scan corresponds to $2.7 \mathrm{GW} / \mathrm{cm}^{2}$ while the $28 \mathrm{~J} / \mathrm{cm}^{2}$ peak fluence of the $23 \mathrm{~ns} \mathrm{XeF}$ scan corresponds to $1.2 \mathrm{GW} / \mathrm{cm}^{2}$. A fluence of $62 \mathrm{~J} / \mathrm{cm}^{2}$ would be needed to achieve 2.7 $\mathrm{GW} / \mathrm{cm}^{2}$. Raster scanning at these fluences is not feasible because experience has shown that surface damage is substantial at fluences above $40 \mathrm{~J} / \mathrm{cm}^{2}$. One can also see the effect of irradiance from the single OSL pulses. For example in the unconditioned quadrant, the $3.3 \mathrm{~J} / \mathrm{cm}^{2}, 0.3 \mathrm{~ns}\left(11 \mathrm{GW} / \mathrm{cm}^{2}\right)$ conditions the $4.7 \mathrm{~J} / \mathrm{cm}^{2}, 0.8 \mathrm{~ns}\left(5.9 \mathrm{GW} / \mathrm{cm}^{2}\right)$ shot which in turn conditions the $12.1 \mathrm{~J} / \mathrm{cm}^{2}, 7.0 \mathrm{~ns}\left(1.7 \mathrm{GW} / \mathrm{cm}^{2}\right)$ shot.

Comparison of the DMS image for the two XeF scan protocols shows a consistently lower pinpoint density for the single pass scan protocol for similar shots. For example, this is evident when comparing the 9.1 and $9.0 \mathrm{~J} / \mathrm{cm}^{2}$ shots for the 11 pass and single pass protocol respectively as well as for other pulse durations. It appears that the single pass protocol is slightly more effective at conditioning for long pulse durations. We cannot say at this time whether this holds for shorter pulse durations.

Continued inspection of the DMS map showed damage occurred in horizontal stripes in the unconditioned and XeF conditioned regions for pulse durations from 0.8 to $1 \mathrm{~ns}$. Nomarski microscopy at up to $200 \mathrm{X}$ showed that this was surface damage corresponding to vacuum chuck marks and was probably due to an overcoated layer of slightly crushed DKDP or other trapped contaminant. This could easily occur during the final diamond turning finishing pass as this 
surface was mounted by vacuum to the chuck holding the part in place. This surface was not incident to the beam during conditioning but was during the OSL damage shots. Typical morphologies are shown in Figure 8.

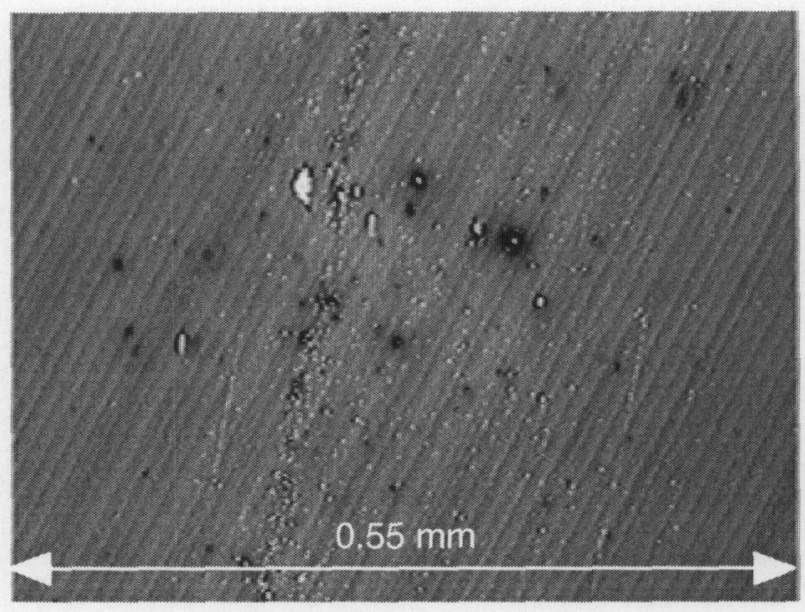

$800 \mathrm{ps}, 4.7 \mathrm{~J} / \mathrm{cm} 2$

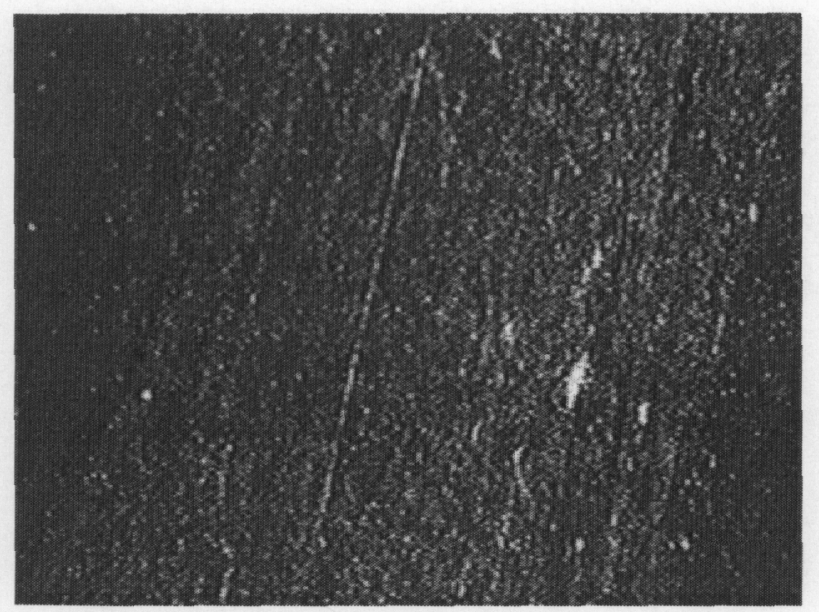

$300 \mathrm{ps}, 3.4 \mathrm{~J} / \mathrm{cm} 2$

Figure 8. Surface damage along vacuum chuck lines. This is likely the result of a slightly crushed layer of DKDP or other contaminant from the diamond turning process. This surface was attached to the vacuum chuck during the last diamond turning pass and was incident to the laser beam during OSL shots.

It is interesting to note that this damage does not appear in the Nd:YAG raster scanned quadrant, nor does it appear in the other regions for OSL shots of $3 \mathrm{~ns}$ or longer. This would indicate that the contamination is most effectively removed by pulses in the $3 \mathrm{~ns}$ regime, and is not activated by pulses longer than $3 \mathrm{~ns}$.

\section{SUMMARY}

This work has shown that significant conditioning can be attained for large aperture DKDP tripler crystals by laser raster scanning with table-top laser systems. Of the two raster scan systems available at LLNL, the $4 \mathrm{~ns} \mathrm{Nd:YAG}$ is much more effective than the $23 \mathrm{~ns} \mathrm{XeF}$ as evidenced by the substantially lower amount of bulk damage. The lack of complete conditioning seen for the $4 \mathrm{~ns}$ scan can, for the most part, be explained by underscanning due to beam wander and fluence fluctuations. In contrast, a similar lack of conditioning cannot be accounted for by beam fluctuations for the $\mathrm{XeF}$ scans as this laser is much more stable and fill factors are much higher. It appears that conditioning depends on irradiance as evidenced by the significant reduction in bulk damage observed when single, short OSL pulses overlap longer pulses. The peak irradiance associated with the $4 \mathrm{~ns}$ scan was $2.7 \mathrm{GW} / \mathrm{cm}^{2}$ while for the $23 \mathrm{~ns}$ scans it was 1.2 $\mathrm{GW} / \mathrm{cm}^{2}$. It would take a fluence exceeding $60 \mathrm{~J} / \mathrm{cm}^{2}$ to achieve the former irradiance with the $23 \mathrm{~ns}$ pulse. This is not feasible because surface damage becomes significant above $40 \mathrm{~J} / \mathrm{cm}^{2}$. Surface damage from either crushed DKDP or overcoated contaminants associated with vacuum chuck lines begins to initiate at pulse durations shorter than $3.2 \mathrm{~ns}$ while pulse durations in the 1 to $3 \mathrm{~ns}$ regime appear to clean the surfaces most effectively. In contrast, $23 \mathrm{~ns}$ pulses were not effective at cleaning the surfaces.

\section{REFERENCES}

1. F. Rainer, F. De Marco, M. C. Staggs, M. R. Kozlowski, L. J. Atherton, L. M. Sheehan, "A historical perspective on fifteen years of laser damage thresholds at LLNL," SPIE Proceedings, Volume 2114, 9-22, 1993

2. M. Runkel, J. DeYoreo, W. Sell and D. Milam, "Laser conditioning study of KDP on the Optical Sciences Laser using large area beams," SPIE Proceedings, Volume 3244, 51-63, 1997

3. M. Staggs, M. Yan, M. Runkel, "Laser raster conditioning of KDP and DKDP crystals using XeCl and Nd:YAG lasers," SPIE Proceedings, Volume 4347, 400-407, 2000 
4. M. Runkel, K. Neeb, M. Staggs, J. Auerbach, A. Burnham, "The results of raster-scan conditioning studies on DKDP triplers using Nd:YAG and excimer lasers," SPIE Proceedings, Volume 4679, 368-383, 2001

5. M. Runkel and M. Nostrand, "An overview of raster scanning for ICF class laser optics," SPIE Proceedings of the Boulder Damage Symposium, 2002

6. C. Stolz, L. Sheehan, S. Maricle, S. Schwartz, "A study of laser conditioning methods of hafnia silica multilayer mirrors," Laser-induced Damage in Optical Materials 1998, SPIE Proceedings, Volume 3578, pp 144-153, 1998

7. F. Rainer, "Mapping and inspection of damage and artifacts in large-scale optics," SPIE Proceedings, Volume 3244, pp 272-281, 1997

8. M. Runkel, A. Burnham, D. Milam, W. Sell, M. Feit and A. Rubenchik, "The results of pulse-scaling experiments on rapid-growth DKDP triplers using the Optical Sciences Laser at $351 \mathrm{~nm}$," SPIE Proceedings, Volume 4347, pp $359-372,2000$

Work performed under the auspices of the U.S. Department of Energy by Lawrence Livermore National Laboratory under Contract No. W-7405-ENG-48. 